\title{
Virtual SEM (VSEM) — Ongoing Development of Teaching Resources
}

\author{
N.H.M. Caldwell, G.C. Martin, A.L.Mitchell, D.M. Holburn, and B.C. Breton
}

Department of Engineering, University of Cambridge, Trumpington Street, Cambridge, CB2 1PZ, United Kingdom

In the last decade, microscopy has been successfully employed by a number of research groups as a means of instilling enthusiasm for science and technology into students of all ages, but particularly school children, e.g. [1]. Remote microscopy (or telemicroscopy) and virtual microscopy (in the form of software simulators) continue to play important roles in these efforts, and also in the teaching and training of new microscopists, e.g. [2 - 4]. The Microscopy \& Microanalysis conference series have become a showcase for such work and associated electronic teaching resources through the regular contributed sessions, e.g. [5].

In our own prior work, we developed a virtual microscope and an interactive encyclopaedia as part of an integrated courseware solution [6]. Initially the encyclopaedia was developed using Adobe (then Macromedia) Director as the authoring tool, due to its ability to combine text, images, simulations, audio and video clips into a coherent whole (see Fig. 1a) [7]. Although used successfully to teach microscopy principles as part of a fourth-year undergraduate engineering course on VLSI, the original encyclopaedia has several drawbacks: the complete package is an executable of significant size which must be downloaded onto a client computer; the executable is self-contained and unable to communicate with other packages; and most importantly, all the content is hard-coded within a design equally customised to supporting that content in terms of layout.

We have now reimplemented the entire encyclopaedia as an Adobe Flash package, following the completion of a laborious content extraction process, which retrieved the individual images, chunks of text and specific animations from the original application. To improve accessibility and maintainability, some material was split into smaller pieces. The new Flash encyclopaedia loads into a browser window in the form of a "master" Flash "movie", which in turn downloads required content from the server (or hard drive) and displays it inside itself. As a user navigates around the encyclopaedia, new data is continually loaded and displayed on-screen (see Fig. 1b).

Every page update involves the use of three XML files: pageList.xml, sequences.xml, and the actual content page itself. pageList.xml lists the unique page ID for each page, its associated title and a directory path to the page content file. sequences.xml lists the sequence of page numbers per section, dividing the encyclopaedia into a number of chapters of related content. This file enables the master Flash movie to enable (or disable) navigation buttons and set their target destinations. Each encyclopaedia page has its own content xml, defining page layout (allowing up to two text boxes and/or two Flash submovies or images to be embedded and positioned in the main movie), the textual content and file paths to external media. The XML files also potentially allow customised information to be made available for the same encyclopaedia page, for instance differentiating according to user knowledge level, e.g. "beginner", "intermediate", and "advanced".

The encyclopaedia's glossary is a searchable list of terms, stored in a distinct xml file. The encyclopaedia uses a Flash feature to dynamically create and embed hyperlinks within a text box. A 
function was written that automatically creates a clickable link out of any glossary term found in the explanatory text. Clicking on a highlighted term triggers a floating dialog box containing the glossary definition.

The advantages of this hybrid XML/Flash approach are the simpler code and the vastly simplified maintenance and upgrading processes, as almost all improvements only require modification of the xml files. There are two disadvantages to the new encyclopaedia, one aesthetic, one practical. The generalised and less granular nature of the new layout means that not all pages are as aesthetically pleasing as their Shockwave counterparts (where individual elements could be precisely positioned). A different design might have included more content panels. Practically, Flash lacks comprehensive support for using HTML tags and character entities (e.g. for mathematical symbols) in imported XML files, so some compromises in formatting and presentation of content were necessary.

The new encyclopaedia provides material on electron guns, focussing and adjusting the electron beam via the column lenses, sample preparation, sample emission, signal detection and image formation. It is already being used to support microscopy teaching within the authors' institution and is being made freely available on the web (see www.virtualsem.com). Having invested the effort in content extraction, ongoing and future work involves creating a microscopy teaching wiki using the material from the encyclopaedia.

\section{References}

[1] G. Fried et al., Microsc. Microanal. 12 (Suppl 2) (2006) 214.

[2] G.S. Casuccio et al., Microsc. Microanal. 8 (Suppl 2), (2002) 1564.

[3] K. Schierbreek et al., Microsc. Microanal. 14 (Suppl 2), (2008) 86

[4] C. Wallace et al., Microsc. Microanal. 14 (Suppl 2), (2008) 858.

[5] J.F. Mansfield, Microsc. Microanal. 14 (Suppl 2), (2008) 876.

[6] G.C. Martin et al., Microsc. Microanal. 13 (Suppl 2) (2007) 1725.

[7] N.H.M. Caldwell et al., Microsc. Microanal. 8 (Suppl 2) (2002) 1566.

[8] G.C. Martin was funded by an EPSRC Studentship, through the Department of Engineering, University of Cambridge, UK
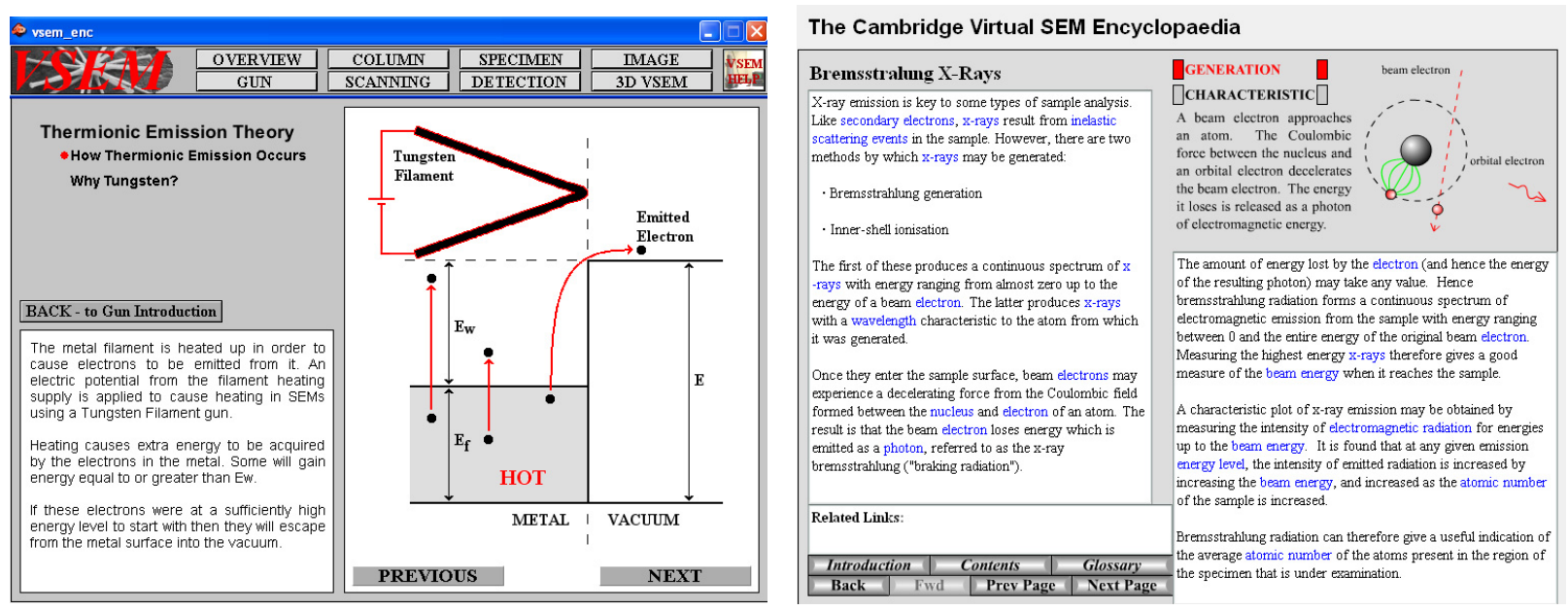

Fig. 1 a) on the left-hand side is a screenshot of the original Director Shockwave VSEM encyclopaedia, and b) on the right-hand side is a screenshot of the new Flash VSEM encyclopaedia. 